\title{
HEAT SHOCK PROTEIN 70 \\ AND NITRIC OXIDE CONCENTRATIONS IN NON-TUMOROUS AND NEOPLASTIC CANINE MAMMARY TISSUES: PRELIMINARY RESULTS - SHORT COMMUNICATION
}

\author{
Marek SZCZUBIAL ${ }^{1}$, Renata URBAN-CHMIEL ${ }^{2}$ and Wojciech ŁoPUSZYŃSKI ${ }^{3 *}$ \\ ${ }^{1}$ Department and Clinic of Animal Reproduction, ${ }^{2}$ Sub-Department of Veterinary \\ Prevention and Avian Diseases, Institute of Biological Bases of Animal Diseases and \\ ${ }^{3}$ Department of Pathological Anatomy, Faculty of Veterinary Medicine, \\ University of Life Sciences, Głęboka 30, 20-612 Lublin, Poland
}

(Received 25 June 2014; accepted 17 February 2015)

\begin{abstract}
The concentrations of heat shock protein 70 (Hsp70) and nitric oxide ions (NO), measured as nitrite, were determined in canine mammary tumours and nontumorous mammary gland tissues. The concentrations of Hsp70 and NO were significantly higher in both benign and malignant tumours than in non-tumorous mammary tissues. Hsp70 concentration decreased with the increase in the grade of histological malignancy. A strong positive correlation was found between the concentrations of Hsp70 and $\mathrm{NO}$ in the benign tumours as well as in grade I and grade II malignant tumours. The results indicate that the process of neoplastic transformation in the canine mammary gland is related to a significant increase in Hsp70 and NO concentration in tumour tissues, and an interdependence between Hsp70 and nitrite ion production can be observed.
\end{abstract}

Key words: Canine mammary tumours, heat shock protein 70 , nitric oxide, mammary gland, female dogs

It has been demonstrated that heat shock protein 70 (Hsp70) can effectively inhibit the apoptosis of tumour cells and promote cancer development (Zorzi and Bonvini, 2011). A high level of Hsp70 expression in human breast cancer has been reported to be correlated with increased proliferation and poor differentiation of tumour cells, lymph node metastases, increased resistance of tumours to chemotherapy, and shortened survival time (Murphy, 2013).

Various studies have shown that nitric oxide (NO) plays an important role in numerous pathological processes including cancer (Xu et al., 2002; Choudhari et al., 2013). The precise function of NO in tumour biology remains unclear, and several studies have demonstrated that NO may have dual, pro- and anti-tumour, effects on cancer (Choudhari et al., 2013).

\footnotetext{
*Corresponding author; E-mail: wojciech.lopuszynski@up.lublin.pl;
} Phone: 0048 (81) 445-6162; Fax: 0048 (81) 445-6166 
Tumours of the canine mammary gland exhibit many similarities to breast cancer in women (Kumaraguruparan et al., 2006; Antuofermo et al., 2007). Although many studies have demonstrated that Hsp70 and NO play an important role in the development and progression of human breast cancer (Choudhari et al., 2013; Murphy, 2013), the role of these indices in the aetiology, pathogenesis and clinical course of canine mammary tumours is not known.

The aim of this study was to evaluate $\mathrm{Hsp} 70$ and $\mathrm{NO}$ concentrations in non-tumorous and neoplastic mammary tissues in female dogs with a view to their potential role in the aetiology, pathogenesis and clinical course of mammary gland tumours.

\section{Materials and methods}

The study was carried out on 34 mammary gland tumours and 10 samples of non-tumorous mammary gland tissue obtained from 34 female dogs that underwent regional mastectomy because of spontaneously occurring mammary tumours. Samples of normal mammary gland tissue collected from non-tumorous glands were removed together with tumours according to a regional mastectomy procedure. All tumours were 3 to $5 \mathrm{~cm}$ in diameter. In order to determine the concentrations of $\mathrm{Hsp} 70$ and $\mathrm{NO}$, fragments (approximately $1.5 \mathrm{~cm} \times 1.5 \mathrm{~cm} \times$ $0.5 \mathrm{~cm}$ in size) of the tumour and non-tumorous mammary gland tissues were collected immediately after mastectomy, then frozen at $-70^{\circ} \mathrm{C}$ and stored until the analysis. The remaining parts of tumour tissues were used for standard histological examination. Tumours were classified according to the World Health Organisation (WHO) criteria for canine mammary neoplasms (Misdorp et al., 1999). Malignant epithelial neoplasms were graded according to the Nottingham method for human breast tumours, adopted for canine mammary tumours (Elston and Ellis, 1998).

Homogenates of mammary tumours and non-tumorous mammary tissues were prepared according to the procedure described by Schmitt et al. (2002), as modified by Salokhe et al. (2006). The concentrations of Hsp70 and NO ions were determined in the homogenates.

The concentration of Hsp70 in the tumours was determined using ELISA kit assays according to the manufacturer's (Cusabio Biotech Co., USA) instructions. The reaction was read with an ELISA reader (680 Bio-Rad, Germany) at $450 \mathrm{~nm}$.

The concentration of NO ions was determined in terms of nitrite ion, as the nitric oxide produced is rapidly converted to nitrite ion (Chui et al., 2004) using Griess reagent (Sigma-Aldrich, Germany). Absorbance was read with a spectrophotometer (Bio-Rad, SmartSpec ${ }^{\mathrm{TM}}$ PLUS, Germany) at $538 \mathrm{~nm}$.

Statistical analysis was performed using the computer program STATISTICA version 10.0. One-way analysis of variance (ANOVA) with Tukey's HSD test was 
used to determine significant differences in the concentrations of Hsp70 and NO between the groups of tissues studied. The correlations between Hsp70 and NO concentration were presented using Pearson's correlation coefficient. Differences at $\mathrm{P}<0.05$ were considered statistically significant.

\section{Results}

Out of the 34 mammary gland tumours studied, 10 were benign ( 6 adenomas, 2 intraductal papillomas, 2 benign mixed tumours) and 24 were malignant (1 carcinoma in situ, 7 complex carcinomas, 10 simple tubulopapillary carcinomas, 2 simple solid carcinomas, 2 anaplastic carcinomas, and 2 spindle-cell carcinomas).

The mean concentration of Hsp70 was significantly higher both in benign and malignant tumours than in non-tumorous mammary gland tissues (Fig. 1A). The highest concentration of Hsp70 was found in malignant tumours. However, no significant difference in the concentration of this protein was found between benign and malignant tumours.

In the group of malignant tumours, the concentration of Hsp70 gradually decreased from grade I tumours to grade III tumours, but a significant difference was found only between these two groups of tumours (Table 1).

Table 1

Mean concentrations of Hsp70 and NO in canine mammary tumours of various grades of histological malignancy

\begin{tabular}{lccc}
\hline Tumour grade & $\begin{array}{c}\text { Number of } \\
\text { tumours }\end{array}$ & $\begin{array}{c}\text { Concentration of Hsp70 } \\
(\mathrm{ng} / \mathrm{mL})\end{array}$ & $\begin{array}{c}\text { Concentration of NO } \\
(\mathrm{mM} / \mathrm{L})\end{array}$ \\
\hline I & 9 & $0.69 \pm 0.2$ & $1.5 \pm 0.6$ \\
II & 9 & $0.64 \pm 0.3$ & $2.1 \pm 0.7$ \\
III & 6 & $0.47 \pm 0.15^{\mathrm{a}}$ & $1.2 \pm 0.3$ \\
\hline
\end{tabular}

${ }^{\mathrm{a}}$ significant differences $(\mathrm{P}<0.05)$ in comparison to grade I tumours

The mean concentration of NO ions was significantly higher both in benign and malignant tumours than in normal mammary gland tissues (Fig. 1B). No significant difference in the concentration of NO ions was found between benign and malignant tumours. In the group of malignant tumours the lowest concentration of nitrite ions was demonstrated in tumours of the highest grade of malignancy; however, the differences were not statistically significant.

Assessment of the correlation between the concentrations of Hsp70 proteins and NO ions in the tissues examined, using Pearson's linear correlation coefficient, demonstrated a very strong positive correlation between these two pa- 
rameters in benign tumours. In the group of malignant epithelial tumours, the correlation between Hsp70 and NO concentration was strong in grade I and grade II tumours, whereas in grade III tumours the correlation was weak (Table 2).

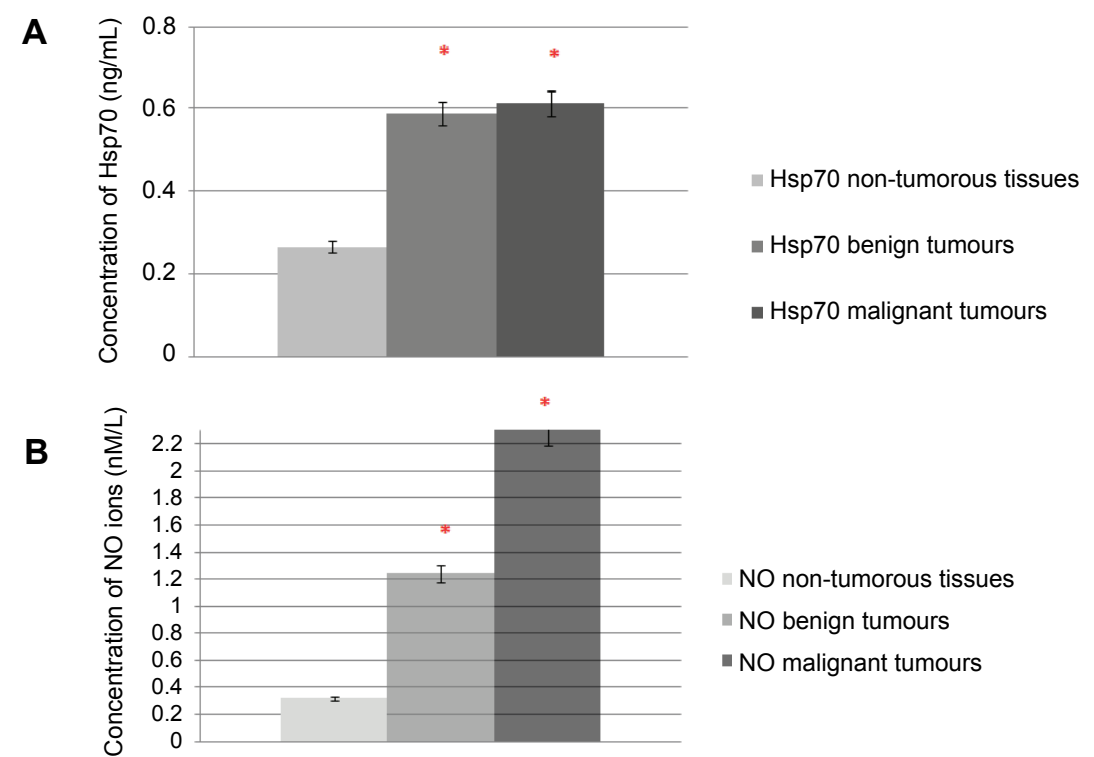

Fig. 1. Mean concentrations of Hsp 70 (A) and $\mathrm{NO}$ (B) in non-tumorous mammary gland tissues and mammary gland tumours. ${ }^{*}$ Significant difference $(\mathrm{P}<0.05)$ in comparison to non-tumorous mammary gland tissues

Table 2

Pearson's correlation coefficients $(r)$ between the concentrations of Hsp70 and NO in non-tumorous mammary gland tissue and mammary gland tumours

\begin{tabular}{lcc}
\hline \multirow{2}{*}{ Examined tissue } & \multicolumn{2}{c}{ Hsp 70 vs. NO } \\
\cline { 2 - 3 } & $r$ & $\mathrm{P}$ \\
\hline Normal tissue & 0.30 & $<0.05$ \\
Benign tumours & 0.96 & $<0.05$ \\
Grade I tumours & 0.67 & $<0.05$ \\
Grade II tumours & 0.56 & $<0.05$ \\
Grade III tumours & 0.27 & $<0.05$ \\
\hline
\end{tabular}

\section{Discussion}

The results of this study show that the levels of Hsp70 and NO are significantly higher in both benign and malignant canine mammary tumours than in non-tumorous tissues of the canine mammary gland. Moreover, the highest con- 
centration of these parameters was observed in malignant tumours. There are some reports that the level of Hsp70 expression is significantly elevated in canine mammary tumours (Kumaraguruparan et al., 2006; Romanucci et al., 2006; Badowska-Kozakiewicz and Malicka, 2012); however, there is no information on the concentration of Hsp70 in canine mammary tumours and non-tumorous mammary gland tissues. This fact makes it impossible to compare our results with those reported by other authors.

In this study we found that the concentration of Hsp70 was high in grade I and grade II tumours and slightly decreased in grade III tumours. Our results support the findings of Badowska-Kozakiewicz and Malicka (2012) who reported that the expression of Hsp70 was the lowest in grade III canine mammary carcinomas.

The present study has shown that both in benign and malignant canine mammary tumours, irrespective of the grade of histological malignancy, the concentration of NO was nearly fourfold higher than in non-tumorous mammary gland tissues. Our findings are consistent with the results of the studies performed by Askar et al. (2009) and Tascene et al. (2011), who demonstrated a significantly higher level of NO in the serum of female dogs with malignant mammary gland tumours as compared to healthy dogs.

In this study we demonstrated the absence of significant differences in the level of NO between benign and malignant tumours, as well as between tumours representing different grades of histological malignancy. This may suggest that every process of neoplastic transformation in the canine mammary gland induces NO production by macrophages and tumour cells, and that the level of this NO production is independent of the histological type and aggressiveness of the tumour. Moreover, the results suggest that the increased level of NO in neoplastic tissues promotes canine mammary tumour development. In this study we demonstrated a positive correlation between the concentrations of Hsp70 and NO in the tissues examined, especially in benign tumours and grade I and grade II malignant tumours, which may indicate an interdependence of Hsp70 and nitrite ion production in canine mammary gland tumours. This is consistent with the findings of a recent study by Song et al. (2013) who showed that apoptotic cells overexpressing Hsp70 induced a marked NO production in macrophages. This suggests that Hsp70 plays an important role both in the up-regulation of NO production and in the host's anti-tumour immunity.

In conclusion, the present study has demonstrated that the process of neoplastic transformation in the canine mammary gland is associated with a significant increase in Hsp70 and NO concentrations in tumour tissues, and an interdependence between Hsp70 and nitrite ion production is observed. These findings suggest that Hsp70 and NO play an important role in the development and progression of canine mammary gland tumours; however, further studies are needed to confirm this. 


\section{References}

Antuofermo, E., Miller, M. A., Pirino, S., Xie, J., Badve, S. and Mohammed, S. (2007): Spontaneous mammary intraepithelial lesions in dogs - a model of breast cancer. Cancer Epidemiol. Biomarkers Prev. 16, 2247-2256.

Askar, T. K., Salmanoglu, B., Salmanoglu, R., Erkal, N. and Beskaya, A. (2009): Changes in the oxidative status and serum trace element levels in dogs with mammary tumours. Acta Vet. (Beograd) 59, 405-411.

Badowska-Kozakiewicz, A. M. and Malicka, E. (2012): Immunohistochemical evaluation of expression of heat shock proteins HSP70 and HSP90 in mammary gland neoplasms in bitches. Polish J. Vet. Sci. 15, 209-214.

Choudhari, S. K., Chaudhary, M., Bagde, S., Gadbail, A. R. and Joshi, V. (2013): Nitric oxide and cancer: a review. World J. Surg. Oncol. 11, 118-129.

Chui, C. H., Tang, J. C., Lau, F. Y., Teo, I. T., Yau, M. Y., Wong, R. S., Cheng, G. Y., Ho, S. K., Leung, T. W., Hui, K. S., Wong, M. M., Fatima, S., Cheng, C. H., Cheung, F., Tan, W. Q., Chow, L. M., Guo, D. and Chan, A. S. (2004): Gleditsia sinensis fruit extract induced growth inhibition involves basic fibroblast growth factor and nitric oxide. Int. J. Mol. Med. 13, 169-173.

Elston, C. W. and Ellis, I. O. (1998): Assessment of histological grade. In: Elston, C. W. and Ellis, I. O. (eds) The Breast. Volume 13. Churchill Livingstone, Edinburgh, New York. pp. 356-384.

Kumaraguruparan, R., Karunagaran, D., Balachandran, Ch., Manohar, B. M. and Nagini, S. (2006): Of humans and canines: A comparative evaluation of heat shock and apoptosis-associated proteins in mammary tumors. Clin. Chim. Acta 365, 168-176.

Misdorp, W., Else, R. W., Hellmen, E. and Lipscomb, T. P. (1999): Histological classification of mammary tumours of the dog and the cat. In: Schuman, F. Y. (ed.) WHO International Histological Classification of Tumors of Domestic Animals. Volume 7. Armed Forces Institute of Pathology, Washington, D.C. pp. 18-25.

Murphy, M. E. (2013): The HSP70 family and cancer. Carcinogenesis 34, 1181-1188.

Romanucci, M., Marinelli, A., Sarli, G. and Della Salda, L. (2006): Heat shock proteins expression in canine malignant mammary tumours. BMC Cancer 6, 171-183.

Salokhe, S., Sarkar, A., Kulkarni, A., Mukherjee, S. and Pal, J. K. (2006): Flufenoxuron, an acylurea insect growth regulator, alters development of Tribolium castaneum (Herbst) (Coleoptera; Tenebrionidae) by modulating levels of chitin, soluble protein content and HSP70 and p34cde2 in the larval tissues. Pestic. Biochem. Physiol. 85, 84-90.

Schmitt, J. P., Schunkert, H., Birbaum, D. E. and Aebert, H. (2002): Kinetics of heat shock protein synthesis in the human heart after cold cardioplegic arrest. Eur. J. Cardiothorac. Surg. 22, 415-470.

Song, S., Zhou, F., Chen, W. R. and Xing, D. (2013): PDT-induced HSP70 externalization up-regulates NO production via TLR2 signal pathway in macrophages. FEBS Lett. 587, 128-135.

Tascene, N., Kismali, G., Vura, S., Karagul, H. and Sel, T. (2011): Serum neopterin, sialic acid and nitric oxide levels in dogs with malignant mammary tumours. Vet. Comp. Oncol. 10, 155-159.

Xu, W., Liu, L. Z., Loizidou, M., Ahmed, M. and Charles, I. G. (2002): The role of nitric oxide in cancer. Cell Res. 12, 311-320.

Zorzi, E. and Bonvini, P. (2011): Inducible Hsp 70 in the regulation of cancer cell survival: analysis of chaperone induction, expression and activity. Cancer 3, 3921-3956. 